\title{
Comparative Effectiveness of Vedolizumab vs. Infliximab Induction Therapy in Ulcerative Colitis: Experience of a Real-World Cohort at a Tertiary Inflammatory Bowel Disease Center
}

\author{
Chaitanya Allamneni ${ }^{\mathrm{a}, \mathrm{f}}$, Krishna Venkata ${ }^{\mathrm{b}}$, Huifeng Yun ${ }^{\mathrm{c}}$, Fenglong Xie ${ }^{\mathrm{c}}$, \\ Lindsey DeLoach ${ }^{\mathrm{d}}$, Talha A. Malik
}

\begin{abstract}
Background: Vedolizumab (VDZ), an adhesion molecule inhibitor and infliximab (IFX), a tumor necrosis factor (TNF) blocker, are both approved as first-line induction agents in moderately to severely active ulcerative colitis (UC). However, there are no head-to-head studies comparing the relative effectiveness of the two agents. Here we provide a real-world comparison of these two agents.
\end{abstract}

Methods: We conducted an ambidirectional cohort study of adult UC patients seen at our tertiary inflammatory bowel disease (IBD) center from 2012 to 2017 . Each patient had moderately to severely active UC via partial Mayo score and was induced with IFX or VDZ. They were followed until assessment of clinical response. Poisson regression was used to calculate clinical response rates and rate ratios.

Results: Of 59 patients who met inclusion criteria, 27 and 32 patients were induced with IFX and VDZ, respectively. Totally, 18/27 (66.7\%) patients induced with IFX vs. 24/32 (78.1\%) patients induced with VDZ were clinical responders. Response rates per 100 person-weeks (PW) were similar for VDZ (5.21) and IFX (5.38). The effectiveness in terms of induction of clinical response (incidence rate ratio, IRR) was not statistically significant for VDZ vs. IFX (IRR 0.97, 95\% confidence interval (CI) 0.53 - 1.77). Among TNF blocker naive patients,

Manuscript submitted November 11, 2017, accepted November 29, 2017

${ }^{a}$ Department of Medicine, University of Alabama at Birmingham, Birmingham, AL 35294, USA

${ }^{b}$ Department of Medicine, University of Alabama at Birmingham, Montgomery, AL 36116, USA

'Department of Epidemiology, University of Alabama at Birmingham, Birmingham, AL 35294, USA

dDepartment of Pharmacy, University of Alabama at Birmingham, Birmingham, AL 35249, USA

eDepartment of Medicine-Gastroenterology, University of Alabama at Birmingham, Birmingham, AL 35249, USA

${ }^{f}$ Corresponding Author: Chaitanya Allamneni, Department of Medicine, University of Alabama at Birmingham, 1720 2nd Ave South, BDB 327, Birmingham, AL 35294, USA. Email: callamneni@uabmc.edu

doi: https://doi.org/10.14740/gr934w
IRR was also not statistically significant between VDZ (6.74/100 PW) and IFX (6.48/100 PW) (IRR 1.04, 95\% CI 0.47 - 2.29). Among TNF blocker experienced patients, there was a higher response rate for VDZ (4.52) vs. IFX (2.29) per $100 \mathrm{PW}$, but the IRR did not reveal statistical significance (IRR $1.97,95 \%$ CI 0.45 - 8.63) due to small sample size of TNF blocker experienced patients who received IFX. Five patients developed severe infection or adverse reaction during IFX induction requiring exclusion, whereas no VDZ patients were excluded for this reason.

Conclusions: Our study revealed a higher proportion of patients who responded to VDZ vs. IFX; however when accounting for period between induction and assessment of clinical response, rates of clinical response were similar. A key difference between the two groups was the higher response rate in the VDZ group among TNF blocker experienced patients; however, a larger cohort is needed to further elaborate on this difference. VDZ held its own against IFX and this study strengthens its standing as a first-line agent among TNF blocker naive as well as TNF blocker experienced UC patients.

Keywords: Ulcerative colitis; Vedolizumab; Infliximab; TNF blocker experienced

\section{Introduction}

Ulcerative colitis (UC) is an inflammatory bowel disease (IBD) characterized by inflammation of the colorectal mucosa. Associated symptoms include rectal bleeding, diarrhea, tenesmus, and abdominal pain. The burden of IBD, and particularly UC, is increasing, with prevalence of UC in North America measured at 198.1 - 298.5 cases per 100,000 persons [1].Surgical therapy is typically reserved for severe disease resistant to medical management. Medical treatment modalities for UC include 5-aminosalicylates, corticosteroids, traditional immunosuppressants including thiopurines, and biologics [2].

Biologic therapy, with infliximab (IFX) and vedolizumab (VDZ) as the two primary options in UC, is typically reserved 
for moderately to severely active disease $[3,4]$. Though effective, $40 \%$ of patients fail to respond to IFX induction therapy [5], and due to blocking antibodies to the drug or development of immune pathways, $30-40 \%$ of patients will lose response to IFX during maintenance phase [6]. In addition, serious infections and malignancies are associated with IFX [7]. Thus, there was a need for a biologic with an alternate mechanism and improved safety profile. VDZ, a humanized monoclonal antibody that inhibits $\alpha_{4} \beta_{7}$-integrin on $\mathrm{T}$ cells from binding to the MAdCAM-1 receptor on gut endothelium and thereby prevents the transfer of inflammatory white cells into the intestinal mucosa, has also been found to be effective in inducing clinical remission or response in UC patients with moderately to severely active disease [8, 9]. Due to this, each of these two agents is separately approved as a first-line induction agent in moderately to severely active UC.

In clinical practice, gastroenterologists are often faced with the dilemma of which agent to use first. While rates of response and remission in UC to IFX have been numerically slightly higher than those to VDZ, the safety profile of VDZ has been superior to that of $\operatorname{IFX}[2,5,10]$. Clinical trial data has revealed similar effectiveness of IFX and VDZ in UC patients when given to TNF blocker experienced patients $[2,5]$. To our knowledge, there are no head-to-head comparison trials comparing the relative efficacy or effectiveness of the two agents. Our study, in which we compare the response of induction between IFX and VDZ, will help guide clinicians during the difficult process of prescribing the right biologic.

\section{Materials and Methods}

\section{Study design, patient population, and selection criteria}

We conducted an ambidirectional cohort study comparing the rates of clinical response to induction by VDZ vs. IFX in patients with moderate to severe $\mathrm{UC}$ at University of Alabama at Birmingham (UAB), a tertiary IBD center. The study was ambidirectional in that it contained both retrospective as well as prospective data, with some patients being induced and followed after initiation of data collection. The study population included adult UC patients seen at the UAB IBD center from 2012 to 2017. To be eligible, patients were required to meet the following criteria: 1) 18 years or older at first observation, 2) clinically measured moderately to severely active UC at first observation, defined by Partial Mayo Score of five or above, 3) duration between clinic visit when decision was made to induce with biologic, and subsequent date of induction was less than 12 weeks, 4) were induced with VDZ or IFX, 5) had at least one follow-up visit within $6-24$ weeks after induction. The first observation was defined as clinic visit that led to decision to induce. During induction period, patients were excluded if diagnosed with colorectal or any other cancer, developed any severe infection, had poor or incomplete documentation, underwent any UC related surgery during induction, developed adverse reaction to biologic necessitating cessation, or

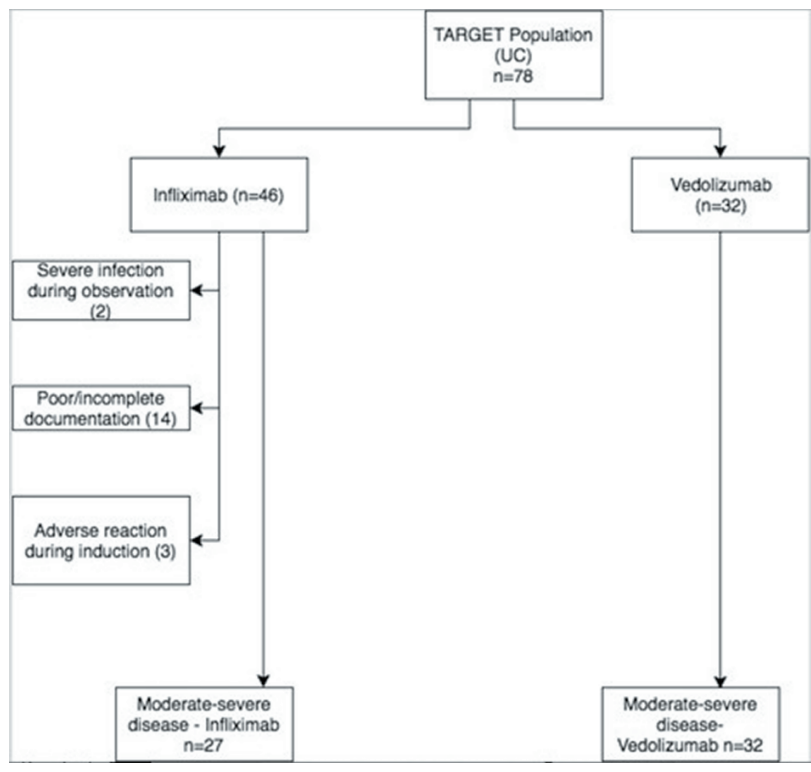

Figure 1. Flowchart of patients who met inclusion/exclusion criteria. UC: ulcerative colitis.

were pregnant (Fig. 1).

The exposure cohort of interest included patients induced with VDZ, and the reference exposure cohort included those induced with IFX. The outcome of interest was clinical response determined by Partial Mayo Score, with response defined as mild disease activity or remission 6 - 24 weeks postinduction. In the Partial Mayo Score, we took a score of 2 - 4 as representing mild disease, and a score of 0 - 1 representing remission. Covariates included age, gender, race, duration of disease, duration of observation, nicotine use, steroid use, thiopurine use, methotrexate use, exposure to TNF-inhibitors, exposure to VDZ, body mass index, disease extent, aminosalicylate use, vitamin D level, proton pump inhibitor (PPI) use, and diabetes mellitus II.

The current study was approved by UAB's Office of Institutional Review Board (IRB).

\section{Data collection and variable definitions}

Data was collected through ambidirectional review of electronic medical records and laboratory results. Factors collected at the time of the first observation in our tertiary center included age, race, gender, duration of UC, disease extent of UC, nicotine use, PPI use, and vitamin D level. Nicotine use was defined as actively smoking at the first observation, PPI use was defined as PPI use at the first observation, and vitamin D was defined as adequate if $25-\mathrm{OH}$ vitamin $\mathrm{D}$ level was over 30 $\mathrm{ng} / \mathrm{mL}$ and inadequate if under $30 \mathrm{ng} / \mathrm{mL}$.

TNF-blocker experienced was defined as prior exposure to one or more TNF-blockers previous to date of induction, including those who did not respond, lost response, or were intolerant. Similarly, VDZ-experienced was defined as prior exposure to VDZ, including those who did not respond, lost response, or were intolerant (Table 1). Steroid use was defined 
Table 1. Summary Statistics (Infliximab vs. Vedolizumab)

\begin{tabular}{|c|c|c|c|}
\hline & Infliximab $(\mathbf{N}=27)$ & Vedolizumab $(\mathbf{N}=32)$ & P-value \\
\hline Age in year, mean (STD) & $42.3(18.5)$ & $54.5(17.9)$ & 0.0135 \\
\hline DOD & $5.8(5.8)$ & $6.6(8.6)$ & 0.6682 \\
\hline Non-Hispanic white & $18(66.7)$ & $21(65.6)$ & \\
\hline Black & $7(25.9)$ & $10(31.2)$ & \\
\hline 0 (male) & $11(40.7)$ & $14(43.8)$ & \\
\hline 1 (female) & $16(59.3)$ & $18(56.2)$ & \\
\hline Nicotine use & $1(3.7)$ & $2(6.3)$ & 1.0000 \\
\hline Steroid use & $8(29.6)$ & $5(15.6)$ & 0.1587 \\
\hline TNF blocker experienced & $6(22.2)$ & $19(59.4)$ & 0.0076 \\
\hline Vedolizumab exp. & $3(11.1)$ & $0(0)$ & 0.0900 \\
\hline Vitamin D def. & $7(25.9)$ & $11(34.4)$ & 0.1059 \\
\hline PPI use & $3(11.1)$ & $6(18.8)$ & 0.4877 \\
\hline BMI & $25.7(5.3)$ & $25.8(5.4)$ & 0.5440 \\
\hline Duration of clinical follow-up after induction, mean (weeks) & 12.38 & 15 & \\
\hline Clinical response & $18(66.7 \%)$ & $25(78.1 \%)$ & 0.3861 \\
\hline
\end{tabular}

STD: standard deviation; 5-ASA: 5-aminosalicylates; PPI: proton pump inhibitor; BMI: body mass index.

as exposure post-induction to parenteral corticosteroids for more than 1 week or rectal, topical, or oral corticosteroids for more than 4 weeks. Aminosalicylate use was defined as use of any rectal/oral mesalamine agent for more than 4 weeks after induction. Thiopurine use was defined as use of azathioprine (AZA) or 6-mercaptopurine (6-MP) for at least 4 weeks post-induction. Methotrexate (MTX) use was defined as use of MTX for at least 4 weeks post-induction. Disease severity was calculated with the Partial Mayo Scoring Index, with a score of 5 or higher indicating moderately active disease. Clinical response was defined as score of 4 or lower, with remission defined as a score of $0-1$.

\section{Statistical analysis}

We conducted descriptive analysis for covariates by exposure groups. T-test was used for continuous variables and Chisquare test was used for categorical variables. Poisson regression models were used to estimate response rates, rate ratios (RR) and 95\% confidence intervals (CI). Bivariate analysis was done to select potential confounders to be included in final model, and covariates with effect size (percent change of adjusted RR from unadjusted RR) equal or greater than $10 \%$ were considered potential confounders. All statistical analyses were conducted using SAS 9.4. P-value $\leq 0.05$ was considered statistically significant.

\section{Results}

Of 78 patients who met initial inclusion criteria, 46 and 32 patients were induced with IFX and VDZ respectively (Fig. 1). Of the 46 induced with IFX, two were excluded due to severe infection, three were excluded due to adverse reaction, and 14 were excluded due to poor or incomplete documentation. None of the VDZ patients had to be further excluded. Three patients were induced with both IFX and VDZ, and thus were included in both categories; however, there was no overlap in personweeks (PW). Totally 18/27 (66.7\%) patients induced with IFX vs. 24/32 (78.1\%) patients induced with VDZ were clinical responders (Table 1,2). When accounting for duration between induction and clinical response assessment, response rates per 100 PW were similar for VDZ (5.21) and IFX (5.38) (Table 2 ). The effectiveness in terms of induction of clinical response (incidence rate ratio, IRR) was not statistically significant for VDZ vs. IFX (IRR 0.97, 95\% CI 0.53 - 1.77). Among TNF blocker naive patients, IRR was also not statistically significant between VDZ (6.74/100 PW) and IFX (6.48/100 PW) (IRR 1.04, 95\% CI 0.47 - 2.29) (Table 3). TNF blocker experienced patients were previously exposed to IFX, golimumab, or adalimumab. Among TNF blocker experienced patients, of 
Table 2. Rate Ratios for Clinical Response

\begin{tabular}{llllll}
\hline & $\begin{array}{l}\text { Proportion with } \\
\text { response }\end{array}$ & $\begin{array}{l}\text { Number with } \\
\text { response }\end{array}$ & $\begin{array}{l}\text { Number of total } \\
\text { person weeks }\end{array}$ & $\begin{array}{l}\text { Response rate } \\
\text { (no. with response/PW)* }\end{array}$ & IRR (95\% CI) \\
\hline Overall & $43 / 59(72.9 \%)$ & 43 & 814 & & 1 \\
Infliximab & $18 / 27(66.7 \%)$ & 18 & 334 & 5.38 & $0.97(0.53-1.77)$ \\
\hline Vedolizumab & $25 / 32(78.1 \%)$ & 25 & 480 & 5.21 & 1.96 \\
\hline
\end{tabular}

*Response rate per 100 person-weeks. IRR: incidence rate ratio; Cl: confidence interval.

Table 3. Rate Ratios for TNF Blocker Naive Patients

\begin{tabular}{llll} 
& Proportion with response & Response rate (number with response/PW)* & IRR (95\% CI) \\
Infliximab & $16 / 21(76.2 \%)$ & 6.48 & 1 \\
Vedolizumab & $10 / 13(76.9 \%)$ & 6.74 & $1.04(0.47-2.29)$ \\
\hline
\end{tabular}

*Response rate per 100 person-weeks. IRR: incidence rate ratio; Cl: confidence interval.

which there were 25 patients totally (6 induced with IFX, 19 with VDZ), there was a higher response rate for VDZ (4.52) vs. IFX (2.29) per 100 PW (Table 4). However, due to small sample size of TNF blocker experienced patients who received IFX, the IRR did not reveal statistical significance (IRR 1.97, $95 \%$ CI $0.45-8.63)$.

\section{Discussion}

Our study revealed an overall numerically higher proportion of patients who responded to VDZ than IFX induction among patients who had moderately to severely active UC at UAB. However, when adjusting for time between induction and assessment of clinical response, the rates of clinical response were similar. In addition, our study revealed a higher clinical response rate to VDZ than IFX in TNF blocker experienced patients. To our knowledge, this is the first study directly comparing these two biologics to one another in a head-to-head approach, although it is not a randomized trial. Prevailing data to this point suggests that IFX has a higher clinical response than VDZ in TNF blocker naive patients, with lower response to both biologics in TNF blocker experienced patients, though similar effectiveness $[2,5]$.

The cardinal trials assessing the efficacy of IFX for induction and maintenance therapy for UC were the Active Ulcerative Colitis Trials 1 and 2 (ACT 1 and 2) [5]. In these multicenter randomized controlled trials, patients with moderately to severely active UC either received infliximab or placebo, and were followed through maintenance therapy until 54 weeks in ACT 1 and 30 weeks in ACT 2. In ACT 1, 69\% of patients who received $5 \mathrm{mg} / \mathrm{kg}$ of infliximab and $61 \%$ of those who received $10 \mathrm{mg} / \mathrm{kg}$ had a clinical response at week 8 , as compared with $37 \%$ of those who received placebo. In ACT 2, 64\% of patients who received $5 \mathrm{mg}$ of infliximab and $69 \%$ of those who received $10 \mathrm{mg}$ had a clinical response at week 8 , as compared with $29 \%$ of those who received placebo. In both studies, patients who received infliximab were more likely to have a clinical response at week 30 . In ACT 1, more patients who received $5 \mathrm{mg}$ or $10 \mathrm{mg}$ of infliximab had a clinical response at week 54 (45\% and 44\%, respectively) than did those who received placebo (20\%). Endoscopic response was also significantly higher in IFX treated patients. However, one limitation in this study performed in 2004 was that all subjects were TNF blocker naive.

The cardinal trial assessing efficacy of VDZ for induction and maintenance for UC was the GEMINI 1 trial, in which two placebo-controlled trials were performed [8]. Patients with moderate to severe UC were induced with VDZ or placebo, and those with response to VDZ were randomly assigned to continue receiving VDZ for maintenance therapy. Response rates at week 6 were $47.1 \%$ and $25.5 \%$ among patients in the VDZ group and placebo group, respectively. At week 52, 41.8\% of patients who continued to receive VDZ every 8 weeks and $44.8 \%$ of patients who continued to receive VDZ every 4 weeks were in clinical remission, as compared with $15.9 \%$ of patients who switched to placebo. Thus, VDZ was more effective than placebo as induction and maintenance therapy.

A key difference between the two groups in our study was the much higher response rate in the VDZ group among TNF blocker experienced patients; however, a larger cohort is needed to further elaborate on this difference. A better safety profile with VDZ than IFX is also supported in this study. Three patients induced with IFX were excluded for severe infection,

Table 4. Rate Ratios for TNF Blocker Experienced Patients

\begin{tabular}{llll}
\hline Clinical cohort & Proportion with response & Response rate (number with response/PW)* & IRR (95\% CI) \\
\hline Infliximab & $2 / 6(33.3 \%)$ & 2.29 & 1 \\
Vedolizumab & $15 / 19(78.9 \%)$ & 4.52 & $1.97(0.45-8.63)$ \\
\hline
\end{tabular}

${ }^{*}$ Response rate per 100 person-weeks. 
and two for an infusion reaction, while none were excluded in the VDZ cohort for these reasons. Severe adverse reactions are uncommon with VDZ, while TNF therapy is an independent predictor of serious infection [7]. Limitations of this study include the relatively small sample size and thus reduced power. In addition, patients were not equally excluded during induction period, with 14 excluded in the IFX group for incomplete documentation, and zero excluded in the VDZ group. This difference could easily introduce bias, but can be explained by the fact that patients were induced with IFX earlier in our study period, when our center was transitioning to electronic medical records, and the system was not as robust. Another limitation of our study is that endoscopic data was not included to assess for endoscopic remission.

Prior studies comparing these two were either meta-analyses or were comparing to placebo $[5,8,11,12]$. The primary advantage of this cohort is that it contains patients from a single institution followed by a select number of IBD specialists. Data was tediously collected, with only the most complete data included, thus strengthening uniformity. Potential drawbacks of VDZ remain its increased cost [13], however research has shown when adjusted for cost per clinical outcome, VDZ had lower cost per patient per year than other TNF blockers, including IFX, adalimumab, and golimumab.

Despite its limitations, this study revealed that VDZ more than held its own against IFX, and given its presumed better safety profile should be considered as a first-line therapy in UC induction among TNF blocker naive as well as TNF blocker experienced patients. Large randomized studies are needed to confirm our current findings regarding the better induction response rate of VDZ in TNF blocker experienced patients with moderately to severely active UC. Future directions should include assessing comparative efficacy of VDZ and IFX in maintenance therapy for UC.

\section{Financial Support}

This study was not funded by any grant and the authors do not have any financial disclosures to make.

\section{References}

1. McLean LP, Shea-Donohue T, Cross RK. Vedolizumab for the treatment of ulcerative colitis and Crohn's disease. Immunotherapy. 2012;4(9):883-898.

2. Bressler B, Marshall JK, Bernstein CN, Bitton A, Jones J, Leontiadis GI, Panaccione R, et al. Clinical practice guidelines for the medical management of nonhospitalized ulcerative colitis: the Toronto consensus. Gastroenterology. 2015;148(5):1035-1058 e1033.

3. Boal Carvalho P, Cotter J. Mucosal healing in ulcerative colitis: a comprehensive review. Drugs. 2017;77(2):159173.

4. Magro F, Gionchetti P, Eliakim R, Ardizzone S, Armuzzi A, Barreiro-de Acosta M, Burisch J, et al. Third european evidence-based consensus on diagnosis and management of ulcerative colitis. part 1: definitions, diagnosis, extraintestinal manifestations, pregnancy, cancer surveillance, surgery, and ileo-anal pouch disorders. J Crohns Colitis. 2017;11(6):649-670.

5. Rutgeerts P, Sandborn WJ, Feagan BG, Reinisch W, Olson A, Johanns J, Travers S, et al. Infliximab for induction and maintenance therapy for ulcerative colitis. N Engl J Med. 2005;353(23):2462-2476.

6. Roda G, Jharap B, Neeraj N, Colombel JF. Loss of Response to Anti-TNFs: Definition, Epidemiology, and Management. Clin Transl Gastroenterol. 2016;7:e135.

7. Lichtenstein GR, Feagan BG, Cohen RD, Salzberg BA, Diamond RH, Price S, Langholff W, et al. Serious infection and mortality in patients with Crohn's disease: more than 5 years of follow-up in the TREAT registry. Am J Gastroenterol. 2012;107(9):1409-1422.

8. Feagan BG, Rutgeerts P, Sands BE, Hanauer S, Colombel JF, Sandborn WJ, Van Assche G, et al. Vedolizumab as induction and maintenance therapy for ulcerative colitis. N Engl J Med. 2013;369(8):699-710.

9. Singh H, Grewal N, Arora E, Kumar H, Kakkar AK. Vedolizumab: A novel anti-integrin drug for treatment of inflammatory bowel disease. J Nat Sci Biol Med. 2016;7(1):4-9.

10. Colombel JF, Sands BE, Rutgeerts P, Sandborn W, Danese S, D'Haens G, Panaccione R, et al. The safety of vedolizumab for ulcerative colitis and Crohn's disease. Gut. 2017;66(5):839-851.

11. Johnston A, Lenti M, O'Connor A, et al. PWE-053 Outcomes of anti-tnf versus vedolizumab therapy for ulcerative colitis: the leeds experience. Gut. 2017;66:A152.

12. Vickers AD, Ainsworth C, Mody R, Bergman A, Ling CS, Medjedovic J, Smyth M. Systematic review with network meta-analysis: comparative efficacy of biologics in the treatment of moderately to severely active ulcerative colitis. PLoS One. 2016;11(10):e0165435.

13. Jansen JP, Mody R, Patel H, Lorenzi M, Ursan I, Alberton $\mathrm{M} .3$ cost per clinical outcomes with biologics for the treatment of moderately to severely active ulcerative colitis. Gastroenterology. 2015;148(4):S-1. 